\title{
ADVANCED NATIONAL AIRSPACE TRAFFIC FLOW MANAGEMENT SIMULATION EXPERIMENTS AND VLIDATION
}

\author{
George Hunter \\ Ben Boisvert \\ Kris Ramamoorthy \\ Sensis Corporation \\ 1700 Dell Avenue \\ Campbell, CA 95008, USA
}

\begin{abstract}
Traffic flow management in the National Airspace is an important problem in our air transportation system. We have developed ProbTFM, a traffic flow management evaluation platform and algorithmic solution. ProbTFM works with existing traffic flow management tools and provides probabilistic data modeling and decision making. ProbTFM forecasts airport and airspace capacity and demand; and airport, airspace, and route congestion. ProbTFM creates a list of high congestion, "critical" flights and recommends delays or reroutes for specific flights. ProbTFM can be used as an evaluation platform for advanced traffic flow management concepts, and to model today's National Airspace System. In this paper we report on validation results and how ProbTFM can be used to understand operational tradeoffs and inform policy decisions.
\end{abstract}

\section{INTRODUCTION}

Air transportation demand has grown significantly in recent decades and is projected to continue to grow in future years. This means the National Airspace System (NAS) will need to implement system improvements if it is to avoid ever increasing congestion.

System improvements range from upgrading the NAS technology and infrastructure to improving the daily decision making that takes place in the NAS. One area of decision making that has tremendous influence on congestion and NAS performance is traffic flow management (TFM).

TFM decision making in the NAS deals with, among other things, real time traffic and weather data. These are used to analyze the current system state as well as forecast the system state in the coming minutes and hours. Both these data sources contain significant levels of uncertainty.

This, as well as other sources of uncertainty, make system forecasting a stochastic problem. This stochastic element is reckoned with in today's NAS, for the most part, subjectively. Operators at practically all levels make intelligent, probabilistic, and in general unrepeatable decisions based on experience and information at hand.

We have developed ProbTFM, an algorithmic TFM tool that objectively makes probabilistic decisions based on stochastic analysis and modeling (Ramamoorthy, 2006).

ProbTFM is a real-time, NAS wide simulation tool for the evaluation of advanced TFM solutions. Our platform can evaluate a wide variety of TFM concepts using realtime system and environmental data. Weather data are provided by WSI.

In addition to the evaluation platform, we have developed an advanced, probabilistic TFM solution in ProbTFM. Our solution is trajectory based. It searches for the steepest gradient in performance improvement per TFM action.

As with most TFM tools, ProbTFM uses trajectory simulation intensively to derive the solution. Therefore the trajectory model is important both for computational performance and for solution accuracy. In this paper we report on validation results for both the ProbTFM trajectory model and for the overall ProbTFM TFM solution. In both cases we find excellent agreement with measured values. Furthermore, our validation results suggest ways that ProbTFM can help inform policy decisions.

\section{TRAJECTORY MODEL VALIDATION}

This section describes our trajectory model and summarizes its validation testing.

\subsection{Trajectory Model Overview}

Trajectory modeling is a core technology required in most advanced air traffic management (ATM) and TFM applications. We use an innovative and extremely efficient, modified equilibrium iteration approach which converges to the exact vertical-plane equilibrium iteratively, accounting for 


\section{Hunter, Boisvert, and Ramamoorthy}

horizontal-plane dynamics (Phillips, 1997). This approach computes the trajectory using variable integration step sizes in appropriate integration variables. The method is fast and retains sufficient fidelity to support conflict detection and resolution when necessary.

The trajectory is computed using a flight plan and aircraft performance data as input. The flight plan specifies waypoints, climb and descent points, nominal cruise speed, and possible altitude constraints. In this method, the vertical profile is specified as a list of segments. Each type of segment has a characteristic path in altitude-velocity space. The equilibrium equations are solved at nodes along each segment. The nodes may be evenly spaced in altitude, velocity, or any convenient parameter which can be mapped to altitude and velocity. Reasonable choices for the integration parameter include altitude, velocity, range, time, flight path angle, and turn angle.

The method solves the exact equilibrium equations iteratively and in the same way for each type of segment, rather than using the exact solution for approximate equations that depend on segment type. This encapsulates the solution in one place and makes it easy to support many type of segments. The method requires an aerodynamic model, a propulsion model, the vertical profile specified by segment type, aircraft configuration, and endpoint, the horizontal profile specified as a series of waypoints, and a weather model which specifies the wind and non standard temperature in four dimensions. No small angle assumptions are made in the equilibrium equations and the trajectories are always continuous.

\subsection{Derivation Using Energy Methods}

The equilibrium equations (Eqns. 1-5) represent the application of Newton's Second Law of Motion in the vertical plane containing the aircraft center of gravity.

$$
\begin{gathered}
\frac{W}{g} \dot{V}=T \cos (\alpha+\varepsilon)-D-W \sin \gamma \\
\dot{h}=V \sin \gamma \\
\frac{W}{g} V \dot{\gamma}=[L+T \sin (\alpha+\varepsilon)] \cos \phi-W \cos \gamma \\
\dot{W}=-f \\
\frac{W}{g} V \dot{\Psi}=[L+T \sin (\alpha+\varepsilon)] \sin \phi
\end{gathered}
$$

These equations are often called the point-mass equations because the aircraft is approximated as a concentrated mass at the center of gravity, as illustrated in Figure 1.

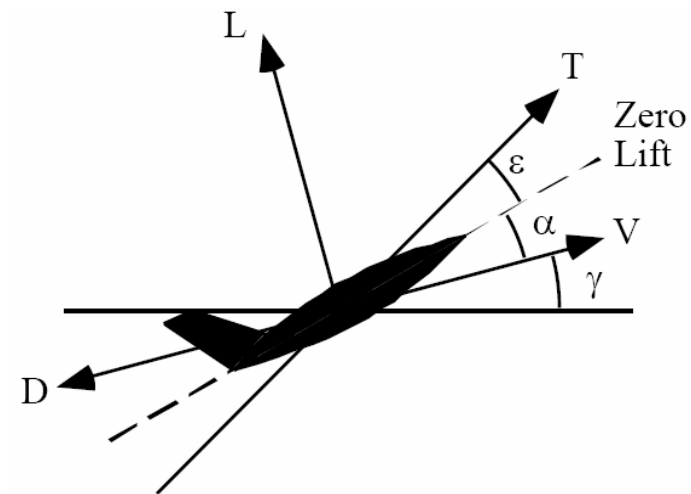

Figure 1: Point mass trajectory model forces and angle definitions.

The energy follows directly as the sum of the potential and kinetic energies:

$$
E=m g h+\frac{1}{2} m V^{2}=W h+\frac{1}{2} \frac{W}{g} V^{2}
$$

Dividing by weight gives the specific energy:

$$
e=h+\frac{V^{2}}{2 g}
$$

Taking the derivative of Equation 7 and substituting from Equations 1 and 2, we have:

$$
\dot{e}=V \frac{T \cos (\alpha+\varepsilon)-D}{W} \equiv P_{s}=V \cdot n_{x}
$$

$P_{s}$ is defined as the specific excess power and $n_{x}$ is the available horizontal acceleration in $g^{\prime}$ s.

\subsection{Equilibrium Iteration and the Horizontal Profile}

In this trajectory model we solve the equilibrium equations iteratively. The state includes weight, lift, thrust, drag, flight path angle, angle of attack, fuel flow, and specific excess energy. The computation proceeds as follows:

1. Compute the next altitude and velocity in the segment.

2. Approximate the new lift coefficient and / or angle of attack.

3. Compute the new drag, thrust, and fuel flow.

4. Compute the new weight using a quadratic formula solution of an implicit equation which uses the average fuel flow and average specific excess power.

5. Compute the new flight path angle.

6. Compute the new lift coefficient.

7. Use the secant method to drive the difference between the guessed lift coefficient at Step 2 and the lift coefficient at Step 6 to zero. This iteration converges rapidly. 


\section{Hunter, Boisvert, and Ramamoorthy}

The input for the horizontal route is a list of waypoints. A waypoint is a latitude-longitude pair. A great circle arc is laid between each pair of waypoints making a leg. An arc is laid in between each pair of legs using a reference true airspeed and minimum bank angle. This breaks the route into a series of alternating turns and straight legs.

With respect to the vertical profile, the difference between a turn and a straight section is that the equilibrium equations must be solved with the correct bank angle and the step size typically needs to be reduced during turns.

The trajectory is integrated using the vertical profile. With each step, the end range is compared against the start range of the next route change. If a step exceeds the start of the next route change, then iteration is performed to find the integration end state that produces the required range. At this point the bank angle and step size are adjusted for the next route change.

The result of an energy step is potentially a new true airspeed. The route defines the true course of the flight. The great circle through the last two points defines the true course direction. The wind triangle is then used to compute the ground speed and heading. The new ground speed and delta time are used to update the range which is then used to update the position using the route.

\subsection{Flight Range and Time Predictions}

We have validated our point mass trajectory model using a variety of aircraft tracking data. Here we present a comparison of the point mass trajectory simulation predictions and en route radar tracking results taken from May 24, 2007. Figure 2 illustrates the medium convective activity in the late afternoon of that day.

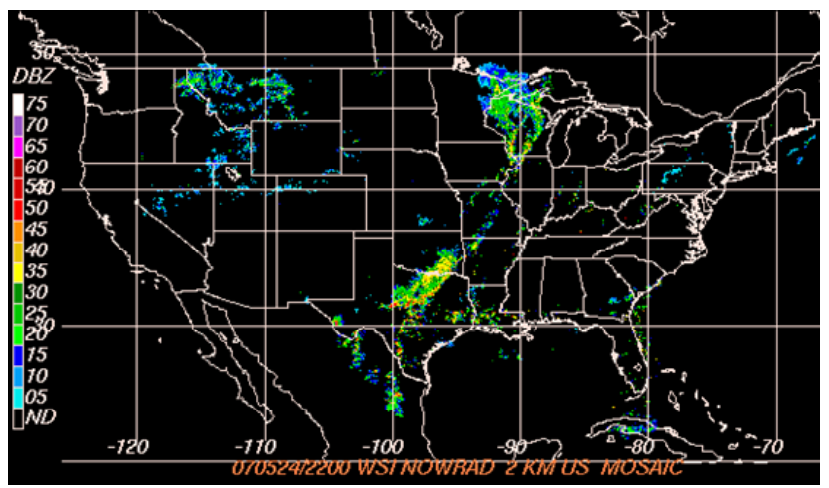

http://www4.ncdc.noaa.gov/cgi-win/wwcgi.dll?WWNEXRAD Images2

Figure 2: May 24, 2007 Afternoon convective weather activity.

Here we compare a randomly selected set of 316 flights, with flight times ranging from 16 to 336 minutes, and mean flight time of 68 minutes. Our objective in this validation test is to evaluate the airborne phase, so we use the first and last piercings of 3,000 ft altitude to define the beginning and ending of the trajectory to be compared. The mean difference between the point mass flight time prediction and the measured flight time (interpolated from the Enhanced Traffic Management System [ETMS] Aircraft Situation Display [ASD] tracking data) is 0.32 minutes with a standard deviation of 11.95 minutes.

As Figure 3 shows, this flight time prediction error is highly correlated with the flight range prediction error. The correlation coefficient is 0.84 .

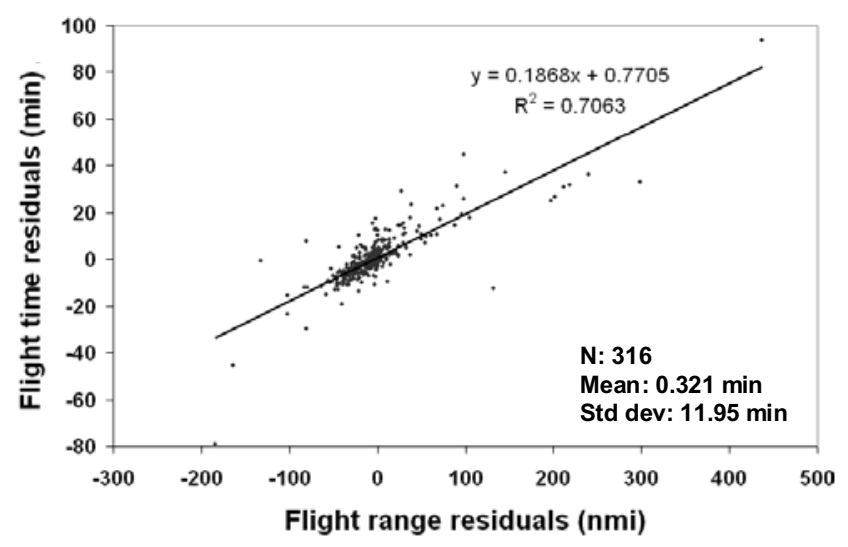

Figure 3: Trajectory prediction flight time error is highly correlated with flight range error. Residuals are computed as the simulated quantity minus the measured quantity.

The mean flight range prediction error is $-2.41 \mathrm{nmi}$, with a standard deviation of $53.75 \mathrm{nmi}$. Deviations in the flight range may arise from a variety of sources. An obvious source of this deviation is the differences in the intended route from that described in the flight plan. This may arise due to pilot's obtaining direct-to vectors to shorten the path, air traffic control delay vectors, weather vectoring, and so forth.

\subsection{Detrended Flight Time Prediction}

The flight plan route is input to the trajectory model. Therefore flight time deviations, due to route deviations, are not a measure of the trajectory model accuracy. These flight time deviations would not occur if the trajectory model had knowledge of the final intended route.

We detrend the flight time prediction error, shown in Fig. 3, to account for the flight range prediction error. This gives us a better estimate of the inherent flight time prediction error of the point mass trajectory model. Figure 4 plots the detrended flight time prediction error data. The detrended mean flight time error is 0.80 minutes, and the standard deviation is reduced almost by $50 \%$, to 6.51 minutes. This prediction error does not show a significant growing trend with longer flights, and is easily within the accuracy requirements for most NAS-wide or 
regional applications, such as traffic flow management decision support tools.

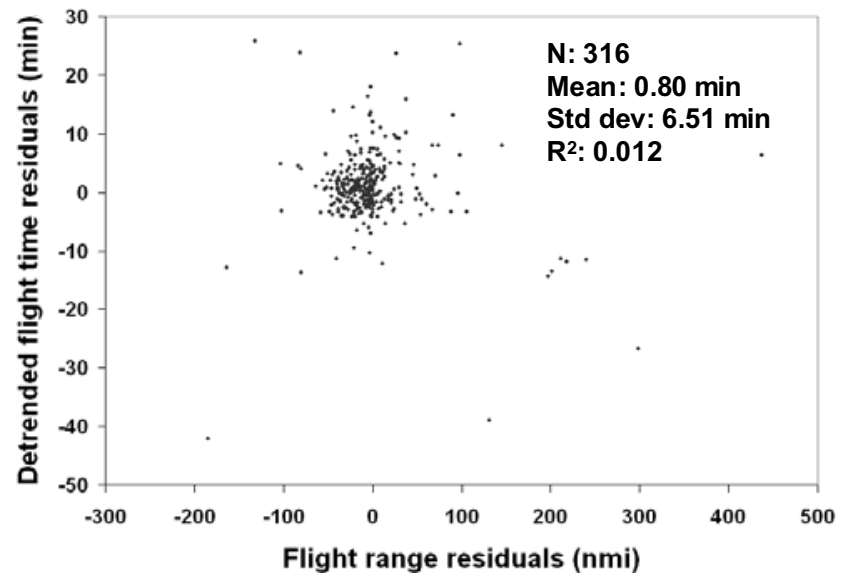

Figure 4: Detrended trajectory prediction flight time error has standard deviation of 6.5 minutes.

\section{TRAFFIC FLOW MANAGEMENT RESULTS AND VALIDATION}

This section presents ProbTFM qualitative and quantitative NAS wide traffic flow management results and validation.

\subsection{System Congestion Versus Delay Tradeoff}

There is, in general, a tradeoff between congestion and delay in finite capacity systems when demand exceeds capacity, such as sometimes occurs the NAS (Wanke, 2005). Consider a scenario where the sustained demand exceeds capacity. If there is no delay, then the traffic throughput is, by definition, identical to demand and congestion occurs (i.e., throughput exceeds capacity). The introduction of delay serves to reduce throughput, and therefore congestion. Eventually, with sufficiently high delay, throughput is reduced to the level of the capacity, and congestion vanishes.

This tradeoff is illustrated in the simple example of a traffic stream, with randomly fluctuating density, transiting an airspace sector. We analyze this tradeoff using a Poisson distribution to model the sector loading, $n$, where $\mu$ is the mean loading.

We define the congestion level as the congestion probability weighted by the magnitude of congestion. For instance, if the probability that a sector is loaded in excess of capacity by two operations is 0.1 , then the corresponding congestion level is 0.2 .

We use hypothetical traffic streams to evaluate the non dimensional mean flight delay and overall congestion level, for this analytical case. Figure 5 plots the results.

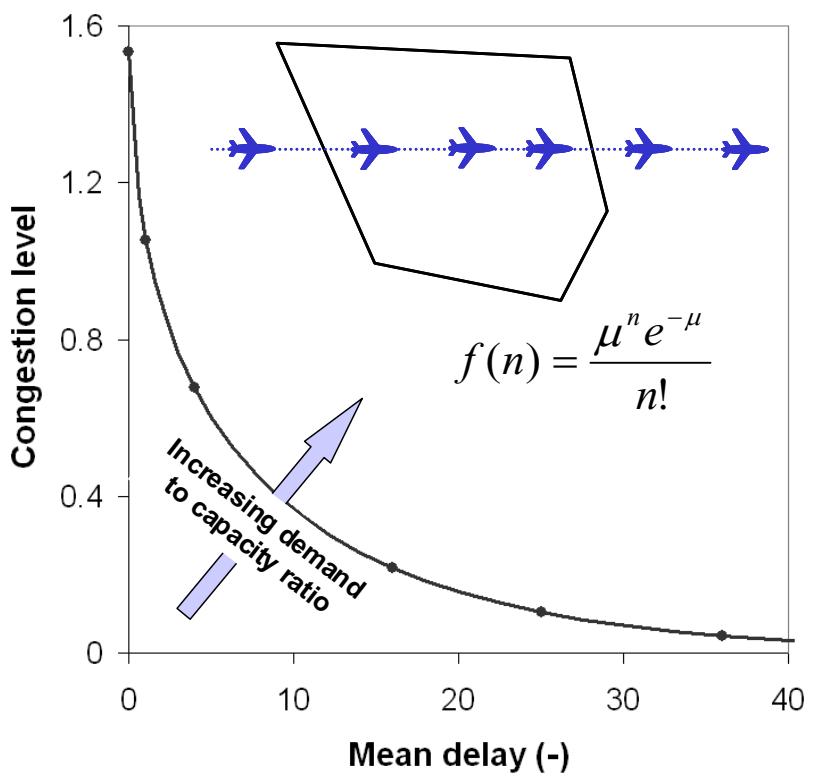

Figure 5: Simple one dimensional illustration of congestion versus delay tradeoff in an airspace sector.

Figure 5 illustrates the tradeoff between mean delay and the frequency of congestion events. Low congestion comes at the cost if high delay, and likewise low delay comes at the cost if high congestion. Figure 5 also indicates the cost of a zero-tolerance policy for congestion. Assuring no congestion comes at the cost of higher delay in this random traffic stream example.

As illustrated in Fig. 5, the tradeoff curve shifts away from the origin with increasing demand or reducing capacity, and toward the origin with decreasing demand or increasing capacity. Therefore a particular congestion versus delay tradeoff exists for a given scenario, and it shifts with changing demand or capacity, for example due to weather or due to the introduction of technology or infrastructure improvements that increase capacity.

Our first aggregate, NAS-wide validation test for ProbTFM is a qualitative one. We evaluate the aggregate, NAS-wide mean flight delay imposed by ProbTFM in its TFM solution, and compare to the aggregate, NAS-wide congestion. Our congestion metric is evaluated, after simulation completion, as the total number of congestion events (each occurring over a 15 minute time interval), weighted by the level of congestion (i.e., the number of operations that the throughput exceeds congestion).

ProbTFM operates on a NAS-wide level. Compared to the single sector analytical solution in Fig. 5, ProbTFM is multi-dimensional and nonlinear. So we do not expect to see an identical congestion versus delay tradeoff in the ProbTFM results. But when the NAS is overloaded, this fundamental tradeoff should be apparent.

We explore this relationship on a NAS wide basis with controlled traffic flows, using ProbTFM to solve the TFM 
problem in a variety of historical NAS days. For instance, Fig. 6 illustrates the weather on Jan. 7, 2007, a Sunday.

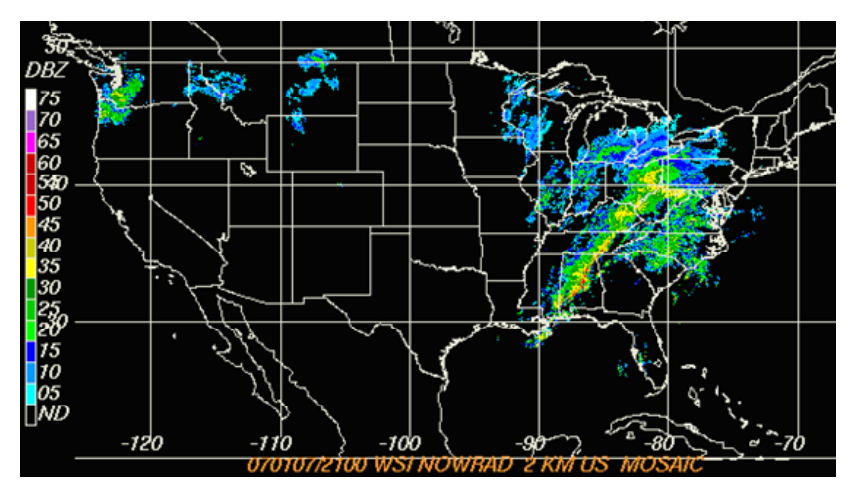

http://www4.ncdc.noaa.gov/cgi-win/wwcgi.dll?WWNEXRAD Images2

Figure 6: Jan. 7, 2007 Convective weather activity.

We vary the congestion sensitivity and measure the resulting mean flight delay and congestion level. Figure 7 shows our results for a series of runs, using traffic schedule and weather data derived from Jan. 7, 2007 archived data. Figure 7 shows a tradeoff between the congestion level and flight delay similar to that of our analytical case in Fig. 5.

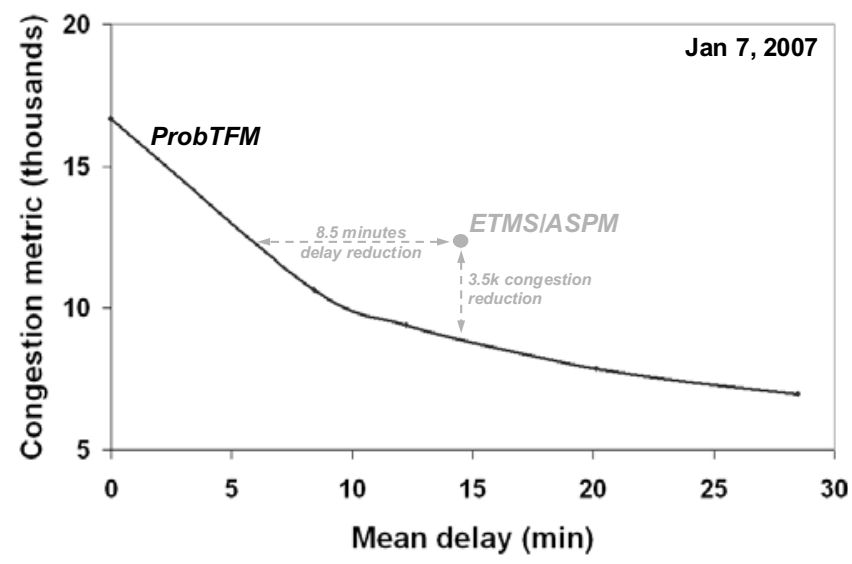

Figure 7: ProbTFM aggregate NAS-wide congestion and delay results for Jan. 7, 2007 simulation Actual data measured using ETMS/ASD traffic data, and ASPM delay data are shown in gray for comparison.

The Fig. 7 tradeoff shows the expected tradeoff between congestion and delay in the ProbTFM results. The NAS congestion is inversely proportional to delay. The relationship shows a more pronounced knee in the curve, compared to the Fig. 5 analytical result.

The Fig. 7 results are typical. In our ProbTFM testing we always see the congestion versus delay tradeoff. Figure 8 shows typical ProbTFM results for NAS days with at least moderate weather.

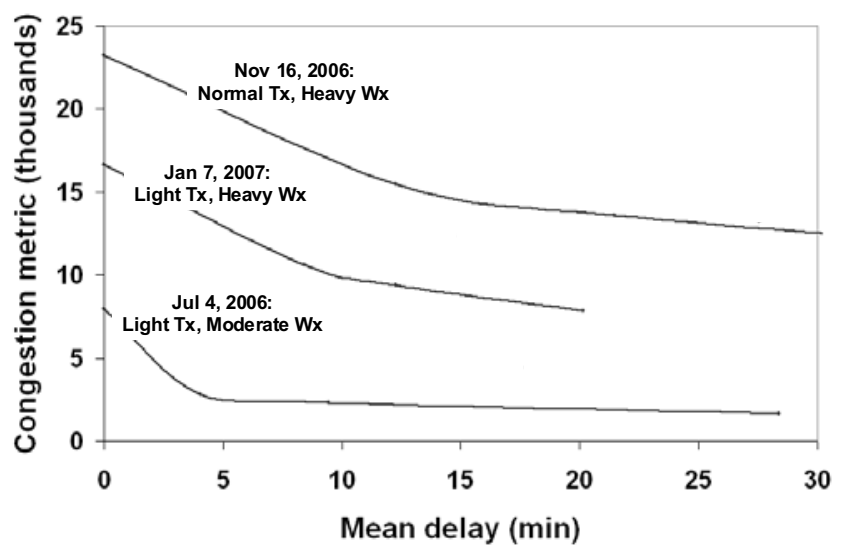

Figure 8: ProbTFM aggregate NAS-wide results for three historical days with increasing levels of traffic and weather.

The Fig. 8 results also validate the behavior of ProbTFM over a range of different NAS days. As illustrated in Fig. 5, the congestion versus delay tradeoff curve should shift away from the origin as the demand-tocapacity ratio increases. As expected, the ProbTFM results in Fig. 8 show increasing shift away from the origin as traffic and/or weather levels increase.

\subsection{System Performance Validation}

We next validate the ProbTFM system-wide results by comparing the ProbTFM congestion versus delay tradeoff with the measured values. We measure the actual congestion metric using the ETMS/ASD traffic data, and obtain an estimate of the flight delay from ASPM. Figure 9 shows the measured values, with arrows pointing to the corresponding ProbTFM simulated curves.

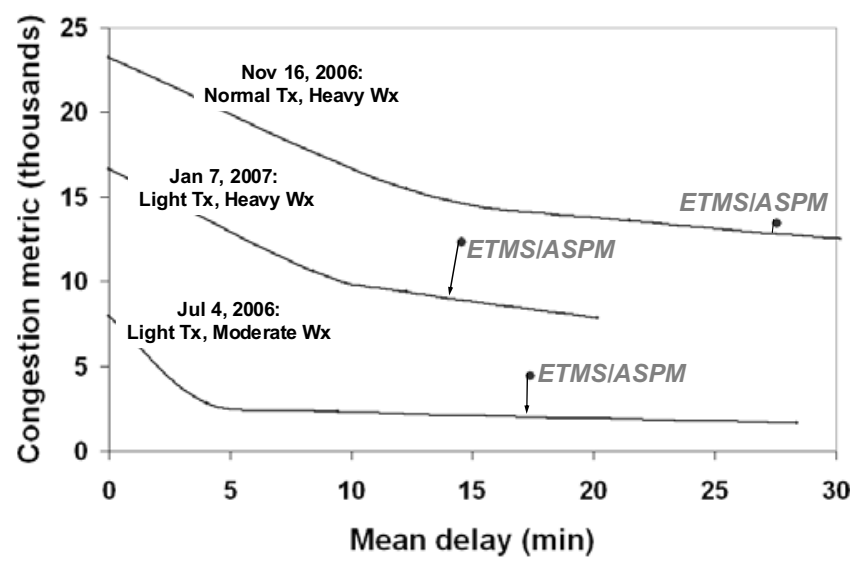

Figure 9: ProbTFM aggregate NAS-wide results for three historical days with data points representing measured congestion and delay values. 


\section{Hunter, Boisvert, and Ramamoorthy}

We have tested several more historical days in addition to the three shown in Fig. 9. As suggested in Fig. 9, we consistently find that the ProbTFM results follow the observed trend in the ETMS/ASPM data. For historical days with high congestion and/or high delay, ProbTFM produces a higher tradeoff curve, with higher congestion and delay. Likewise, for historical days with lower congestion and/or low delay, ProbTFM produces a lower tradeoff curve, with lower congestion and delay.

\section{DISCUSSION AND CONCLUSIONS}

In this paper we show validation data for both the ProbTFM trajectory model and overall aggregate performance. In particular, our comparison of the ProbTFM trajectory model with tracking data shows a mean flight time error of less than one minute with a standard deviation of 6.5 minutes. This accuracy is sufficient to support NAS wide TFM applications.

Concerning ProbTFM aggregate performance, we qualitatively validated the shape of the congestion versus delay curve produced by ProbTFM, and we validated the shifting of the curve with changes to the NAS-wide demand-to-capacity ration. We also validated that the ProbTFM congestion versus delay curves correlate with the measured NAS performance.

These validations suggest that ProbTFM can be used to simulate the NAS performance. A major advantage of ProbTFM is that successive runs, with varying congestion sensitivity, can be used to generate the congestion versus delay curve for a given NAS day. So rather than merely reviewing measured NAS performance, ProbTFM provides curves that indicate the full range of possible operating points where the NAS could have performed. This information is useful to policy makers.

That is, the congestion versus delay tradeoff curves in Fig. 9 suggest that, without technology or infrastructure improvements, the NAS performance can vary, from the observed operating points, along the tradeoff direction to different congestion and delay settings. In this sense, these congestion versus delay tradeoff curves represent contours of constant NAS performance. In this view, moving along the tradeoff curve does not represent a change in performance, but rather a change in policy. On the other hand, moving orthogonal to the tradeoff curve represents a change in performance, with performance improving in the direction toward the origin.

Figure 10 shows the measured delay and congestion for seven historical days, with various traffic and weather conditions. The Fig. 9 performance contours are shown in gray for reference. As expected the NAS measured performance moves away from the origin as traffic and/or weather level increase. But the ProbTFM performance contours suggest that significant delay reduction is possible at the cost of minimal congestion increase.

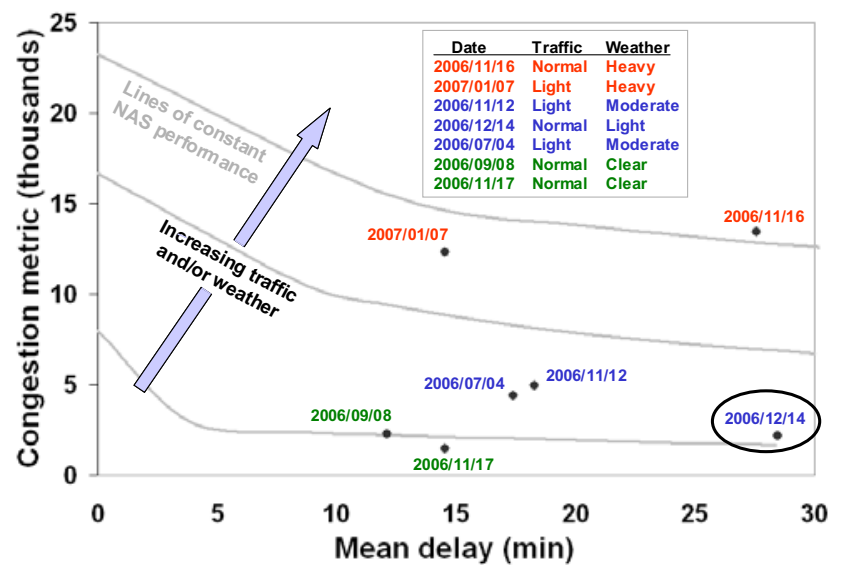

Figure 10: ProbTFM performance contours suggest NAS policy changes.

Delay reduction with only minimal congestion increase is possible when the NAS operating point is to the right of the knee in the performance contour. This is the case for several of the days plotted in Fig. 10, and particularly so for Dec. 14, 2006 (circled on the plot).

The performance contours indicate that the NAS performance on Dec. 14, 2006 was reasonable. There was light weather and normal traffic, and the congestion and delay observed for that day fall onto a contour that is similar to that of other days with similar conditions. Also, the Dec. 14, 2006 operating point is only slightly above the ProbTFM simulated performance contour for that day (not shown).

But the Dec. 14, 2006 operating point, though close to the simulated performance contour, is far from the knee in the curve. The operating point is in the flat part of the curve, and this suggests that substantial delay reduction is possible with only minimal congestion increase. In the case of Dec. 14, 2006, a mean delay reduction of up to 15-20 minutes may be possible.

The policy change to shift days, such as Dec. 14, 2006 , toward the knee in the performance contour, is to accept slightly more congestion (easily within the NAS tolerance) in order to reduce delay significantly. This means changes to the TFM decision making regarding how weather and other system uncertainties are handled. The specific changes required, however, are complicated because the NAS policy is not currently repeatable. TFM decision making is determined by a large number of decisions made by a variety of operators. Sometimes these decisions are made objectively according to recognized guidelines. But often they are unrepeatable, subjective decisions made in the absence of specific guidelines.

ProbTFM is a prototype TFM tool that provides an objective set of guidelines such that TFM decision making is objective, repeatable, and public. ProbTFM integrates 
weather into TFM/ATM decision making, and it is based on a single, unified weather database. This approach lays the groundwork for a common situational awareness between TFM provider and operators. In a collaborative scheme, ProbTFM could be simultaneously used by operators and provider, thus providing transparency in TFM decision making to operators so they can anticipate problem flights and remedy them so the TFM provider will not need to.

Finally, we note that the ProbTFM performance contours we have generated typically have improved performance compared to the ETMS/ASPM measured congestion and delay values. Figure 9, for instance, shows that two of the three days plotted show substantial delay reductions in the ProbTFM performance compared to the ETMS/ASPM measurements. This suggests that a probabilistic, trajectory-based approach, such as ProbTFM, can provide good performance improvement in today's system.

\section{ACKNOWLEDGMENTS}

The authors acknowledge the contributions and insights from many co-workers, including Jim Phillips, Dr. Fred Wieland, and Dr. Sherry Borener. The authors would also like to acknowledge Mitre CAASD and WSI for their crucial contributions and the staff of Sensis Corporation for their support of this work.

\section{REFERENCES}

Phillips, James D. 1997. An Accurate and Flexible Trajectory Analysis. In World Aviation Congress (SAE Paper 975599), Anaheim, CA, October 13-16.

Ramamoorthy, K., B. Boisvert, and G. Hunter. 2006. A real-time probabilistic traffic flow management evaluation tool. In 25th Digital Avionics Systems Conference, Portland, OR, Oct. 16-19.

Wanke, C., L. Song, S. Zobell, D. Greenbaum, and S. Mulgund. 2005. Probabilistic Congestion Management. In 6th USA / Europe Air Traffic Management $R \& D$ Seminar, Baltimore, MD, June 27-30.

\section{AUTHOR BIOGRAPHIES}

GEORGE HUNTER has over twenty years of aerospace engineering experience including work in the areas of optimal estimation, aviation and meteorology, and air traffic management. He received his B.S and M.S. degrees from the University of Michigan, and Ph.D from the University of Illinois.

BEN BOISVERT has over sixteen years of software engineering experience in the aerospace industry. He has fielded and maintained three oceanic air traffic systems and developed two air traffic simulations. He received his M.S. from the Rochester Institute of Technology.

KRIS RAMAMOORTHY has, over the past three years, performed extensive analysis and simulation of National Airspace System. His interests include nonlinear systems analysis and optimization, and air traffic management. He received M.S. from the State University of New York at Buffalo. 